\title{
Geometric Robust Multimodal Biometric Watermarking Scheme for Copyright Protection of Digital Images
}

\author{
Meenakshi S Arya \\ Deptt of CSE \& ICT \\ JUIT, Waknaghat \\ Solan, HP, India
}

\author{
Rajesh Siddavatam \\ Deptt of CSE \& ICT \\ JUIT, Waknaghat \\ Solan, HP, India
}

\begin{abstract}
This paper proposes a copyright protection system using multimodal biometrics for content protection of digital images. Two biometric traits, one physiological (face) and one behavioral (offline handwritten signature) of the legitimate owner are used as watermarks. These watermarks are embedded at different levels of resolution to the high frequency wavelet coefficients through optimal control of the embedding factor. Experimental evaluation confirms the imperceptibility and robustness of the scheme against a family of typical image distortions functions like Jpeg Compression, filtering etc as well as geometric attacks such as scaling, rotation and combined attacks.
\end{abstract}

\section{General Terms}

Pattern Recognition, Digital Rights Management, Copyright Protection.

\section{Keywords}

multimodal biometrics, lifting wavelet transform, biorthogonal wavelets, structural similarity index measure.

\section{INTRODUCTION}

"A picture is worth a thousand words", this famous anecdote specifies the amount of information that a picture is capable of conveying. Technological advancements in acquisition, processing and storage have made it possible to capture, store and disseminate life-like images, at the same time raised the threat of manipulation, altering or permanent tampering of this data. There is hence a need felt to provide an authentication data within this image data to ensure the originality and copyright protection. This process of adding small distortions to image by exploiting the redundancy in image data and the fact that Human Visual System is also insensitive to such distortions is called watermarking. Watermarking is the best remedy for protecting and preserving the rightful ownership of any useful data. The image coefficients can be perturbed either spatially or after the image has been transformed in frequency domain. While the former technique involves random selection of subsets of host image for the purpose of watermark insertion, the latter inserts the same into the coefficients of the transformed image. The most common image transforms used are Discrete Fourier Transform (DFT), Discrete Cosine Transform (DCT) or Discrete Wavelet Transform (DWT).

Biometric watermarking is a watermarking technique in which a biometric trait of a person like fingerprint, face, voice or iris is used as the watermark. As biometric traits are unique to an individual, their use as watermarks offer an enhanced access control mechanism, and serve as stronger deterrents to piracy.

This paper proposes a copyright protection using multimodal biometrics for content protection of digital images. Two biometric traits, one physiological (face) and one behavioral (offline handwritten signature) of the legitimate owner are used as watermarks. These watermarks are embedded at different levels of resolution to the high frequency wavelet coefficients through optimal control of the embedding factor. Experimental evaluation confirms the imperceptibility and robustness of the scheme against a family of typical image distortions functions like Jpeg Compression, filtering, scaling. The rest of this paper is organized as follows. Section II reviews biometric watermarking implementations for image data and the concept of lifting wavelet transform. Section III outlines the proposed algorithm framework. Section IV illustrates the optimization of embedding factor. The experimental results and discussions are presented in Section V. The paper is summed up and concluded in Section VI.

\section{RELATED WORK}

\subsection{Literature review}

Biometric digital watermarking was primarily proposed by A.K.Jain and his research team. Jain and Umut [1] proposed multimedia content protection framework that is based on biometric data of the users. Kundur and Hatzinakos [2] were the pioneers in suggesting a watermarking model using biorthogonal wavelets based on embedding a watermark in detail wavelet coefficients of the host image. The model proposed was robust against numerous signal distortions, however it was non-blind. Yang [3] in his paper simulates under a spread-spectrum watermarking framework where a Gaussian distributed watermark is injected into the largest wavelet coefficients to find the best biorthogonal wavelet filter for multi resolution image watermarking. The performance of seven integer biorthogonal wavelet bases is evaluated and it is observed that the 917-F wavelet provides a substantial edge ${ }^{e c}$ when all detail sub bands are eligible for watermarking. The effect of using even-length and odd length biorthogonal wavelets for watermarking have been discussed in [4] and [5] respectively. Both these techniques were robust against several attacks, but were presented for the sake of detecting the presence of a watermark not for extracting it. M.Vasta[6] presented a novel biometric watermarking algorithm for improving the recognition accuracy and protecting the face and fingerprint images from tampering. He made use of multi resolution DWT to embed face image in a finger print image. V-Support Vector Machine is exploited to enhance the quality of the extracted face image. Low et al. [7] proposed to adaptively fuse Least Significant Bit (LSB) and 
Discrete Wavelet Transform (DWT)-based approaches into a unison framework, which to be known as LSBDWT scheme. The performance of LSB-DWT scheme is validated against simulated frequency and geometric attacks. Namboodiri, Jain [8] presented an LSB-based biometric watermarking scheme where a digital document was spatially watermarked with online handwritten signature. Reddy et al. [9] proposed a method in which the authors used a gray scale logo as watermark. Further, they used the model of Barni et al. [10] to calculate the weight factors for wavelet coefficients of the host image. They extracted watermark from the distorted image by taking into consideration the distortion caused by the attacks. Fig 1 presents a generic biometric watermark extraction and embedding algorithm.

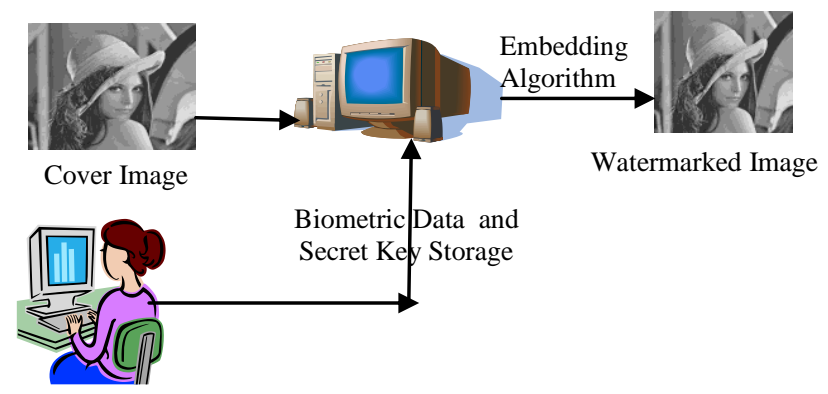

Legitimate owner

(a)

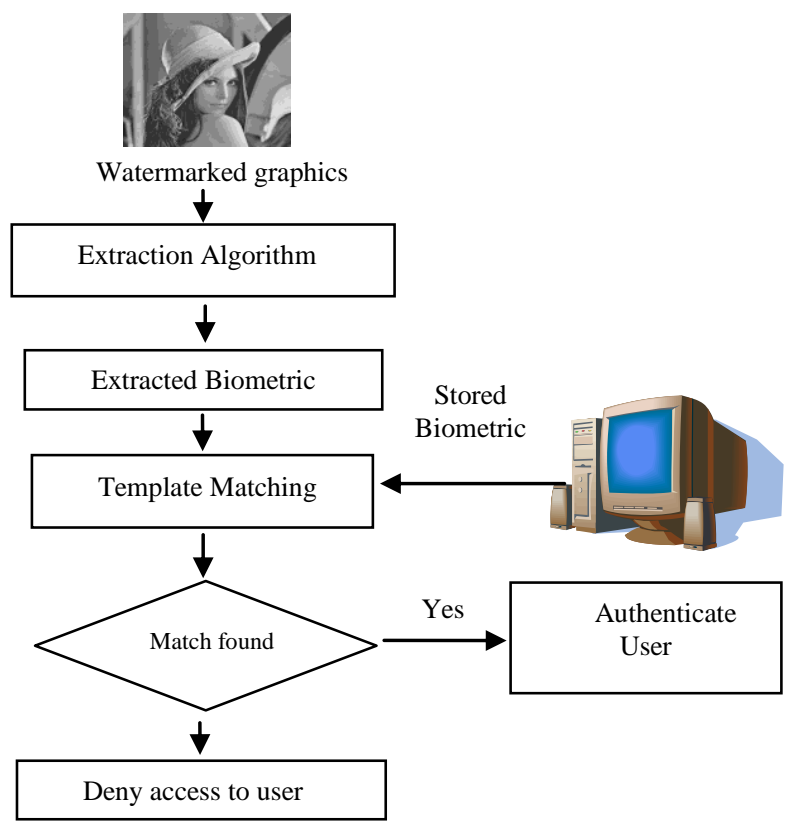

(b)

Fig 1: (a) Generic biometric watermark embedding (b) Generic biometric watermark extraction and authentication.

\subsection{Lifting Wavelet Transform Using Biorthogonal Wavelets}

In the proposed algorithm, lifting scheme proposed by Swelden [11] has been used for multiresolution decomposition of the host image. Computation of LWT can be carried out either using convolution based (filter bank) procedures or lifting based procedures The lifting wavelet transform is widely used in signal processing because of its efficient implementation with low memory and computational complexity [12]. Figure 2 depicts the process of lifting wavelet transform and inverse lifting wavelet transform

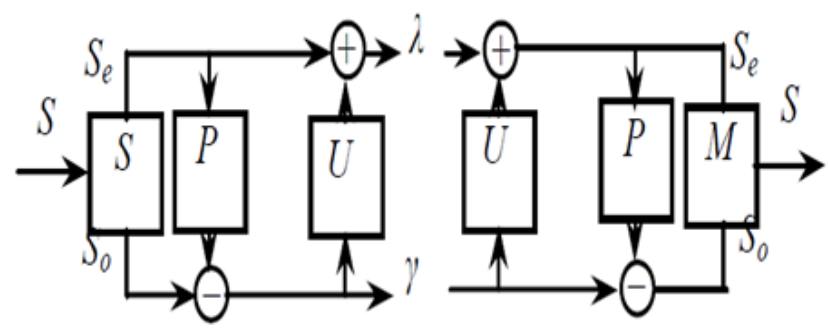

Fig 2: Basic block diagram of lifting wavelet transform

The lifting algorithm consists of three phases: Split, Predict and Update. The 2-D image $f(x, y)$ passes through the following steps to obtain the wavelet coefficients.

- $\quad$ Split: $f_{e}(x, y)=f(x, 2 y)$

$f_{o}(x, y)=f(x, 2 y+1)$

- $\quad$ Predict: $\gamma(x, y)=f_{o}(x, y)-P\left[f_{e}(x, y)\right]$

- Update: $\lambda(x, y)=f_{e}(x, y)+U(x, y)$

The lifting wavelet transform used in the present context has been strengthened by using biorthogonal wavelet transform. A biorthogonal wavelet is a wavelet where the associated wavelet transform is invertible but not necessarily orthogonal. Designing biorthogonal wavelets allows more degrees of freedoms than orthogonal wavelets. One additional degree of freedom is the possibility to construct symmetric wavelet functions [13].

The property of perfect reconstruction and symmetric wavelet functions exist in biorthogonal wavelets because they have two sets of low pass filters (for reconstruction), and high pass filters (for decomposition). Another advantageous property of biorthogonal over orthogonal wavelets is that they have higher embedding capacity if they are used to decompose the image into different channels. In the case of Biorthogonal wavelet, rather than a single scaling function there is a dual scaling function and mother wavelet.

$$
\begin{aligned}
& \phi_{k}^{n}(x)=2^{\frac{n}{2}} \phi\left(2^{n} x-k\right) \\
& \psi_{k}^{n}(x)=2^{\frac{n}{2}} \psi\left(2^{n} x-k\right)
\end{aligned}
$$

The correlation is calculated between the embedded watermarks and the image decomposition coefficients obtained using classical LWT and Biorthogonal LWT. The Biorthogonal LWT provides lower correlation with the embedded watermarks due to the complementary information present in two wavelet systems that offers better directional selectivity compared to classical transform.

\section{PROPOSED METHOD}

In the proposed algorithm, the classic Cox's digital watermarking [13] algorithm concept has been suitably modified to embed two biometric traits i.e. an offline handwritten signature and a face image of the owner of digital image. The host image is decomposed using lifting wavelet transform using biorthogonal wavelets as described in [14] .The watermarks are embedded at different levels of resolution hence providing geometric attack resilience to the proposed algorithm. The biometric images used are gray 
scale images and which are binarized using algorithm described in [15].

\subsection{Watermark Embedding}

The following steps are followed for the watermark embedding.

i. Let $I_{\text {original }}$ be the host image of size $M X N$.

ii. Let $w m_{1}$ and $w m_{2}$ be the signature watermark and face watermark of length $\left(M_{S} X N_{s}\right)$ and $\left(M_{f} X N_{f}\right)$ respectively. The feature vectors are generated after binarization by converting the watermarks into a 1D array of $\operatorname{size}\left(\left(M_{s} * N_{s}\right), 1\right)$ and $\left(\left(M_{f} * N_{f}\right), 1\right)$ resp.

iii. Secret key $K 1$ is entered to generate pseudorandom sequences for signature watermark insertion.

iv. For embedding the signature image, the Lifting Wavelet Transform $I_{l w t(i, j)}$ of the host image is calculated for level 2 decomposition, sub-bands of size $\frac{N}{2^{2}} \times \frac{N}{2^{2}}$ are obtained.

v. The horizontal detailed band is used for watermark insertion as embedding in the approximation band causes more perceptible changes to the watermarked image. The watermark is embedded as follows: random_seq 1 for $i=1$ : length $\left(w_{1}\right)$

$$
\begin{aligned}
& \quad=\operatorname{round}(2 *(\operatorname{rand}(M / 4, N / 4) \\
& \quad-0.5)) ; \\
& \text { if }\left(w m_{1}(i)==0\right)
\end{aligned}
$$

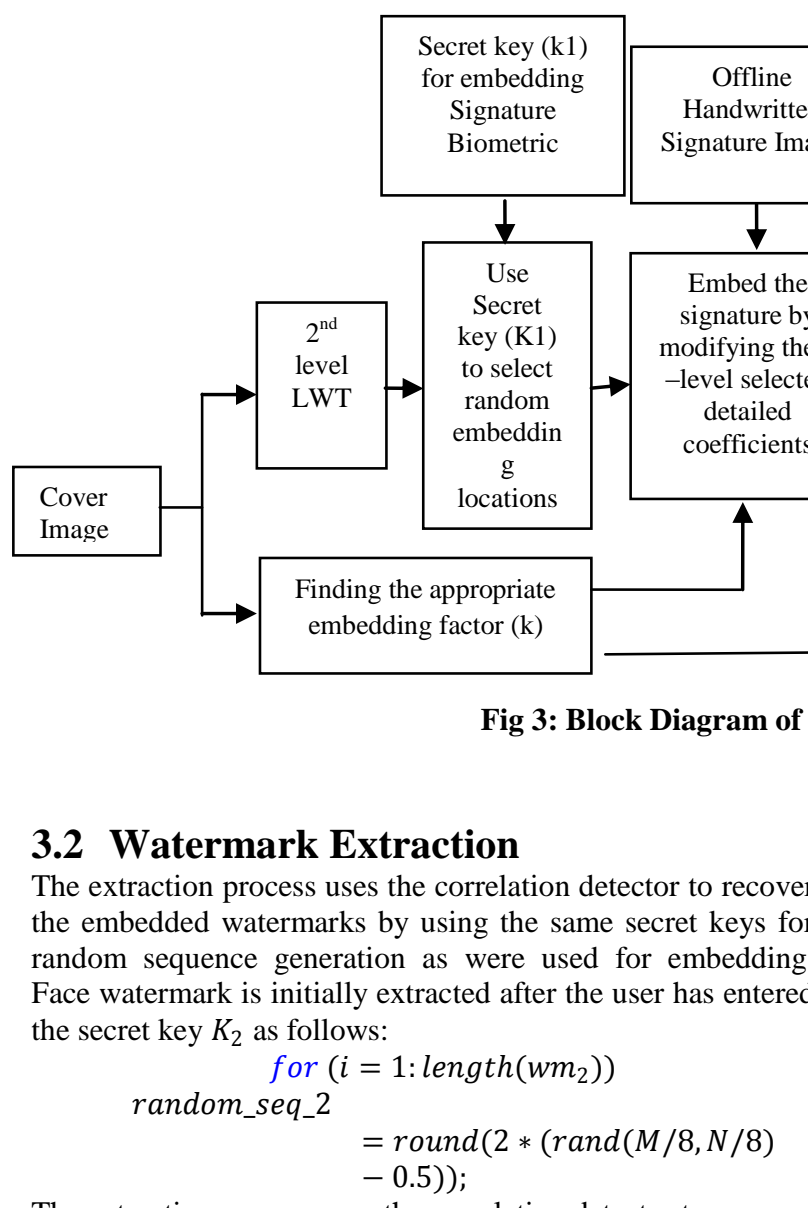

The extraction process uses the correlation detector to recover the embedded watermarks by using the same secret keys for random sequence generation as were used for embedding.

$$
\begin{gathered}
H H_{2}=H H_{2}+k * r a n d o m \_s e q \_1 ; \\
\text { end } \\
\text { end }
\end{gathered}
$$

$k$ is the strength factor used for embedding. Any visible effect, such as blocking, due to manipulation of these wavelet coefficients is controlled using parameter optimization method as described in the subsequent section. Experimentally it has been observed that this factor must be $<1$.

vi. $\quad$ Secret key $K 2$ is entered to generate pseudorandom sequences for face watermark insertion.

vii. For embedding the face image, the Lifting Wavelet Transform $I_{l w t(i, j)}$ of the host image is calculated for level 3 decomposition, sub-bands of size $\frac{N}{2^{3}} \times \frac{N}{2^{3}}$ are obtained.

viii. The level 3 horizontal detailed band is used for embedding the face image as follows:

random_seq_2

$$
\text { for } i=1 \text { : length }\left(w m_{2}\right)
$$

$$
\begin{gathered}
=\operatorname{round}(2 *(\operatorname{rand}(M / 8, N / 8) \\
-0.5)) ; \\
\text { if }\left(w_{2}(i)==0\right) \\
\mathrm{HH}_{3}=\mathrm{HH}_{3}+k * \text { random_seq_2 } \\
\text { end } \\
\text { end }
\end{gathered}
$$

ix. The watermarked image $I_{w m}$ is obtained by applying inverse LWT twice as shown in fig 3 . 
random_seq_1

$$
\begin{aligned}
& =\operatorname{round}(2 *(\operatorname{rand}(M / 4, N / 4) \\
& -0.5))
\end{aligned}
$$

correlation $_{\text {signature }(i)}$

$$
=\operatorname{corr} 2\left(\mathrm{HH}_{2}, \text { random_seq_1 }\right) \text {; }
$$
end

$$
\begin{gathered}
\text { if (correlation_signature }(i) \\
\left.>\text { std }\left(\text { correlation_HH } H_{2}\right)\right) \\
w m_{1}(i)=0 ; \\
\text { else } w_{1}(i)=1 ; \\
\text { end }
\end{gathered}
$$

The same is depicted in fig. 4

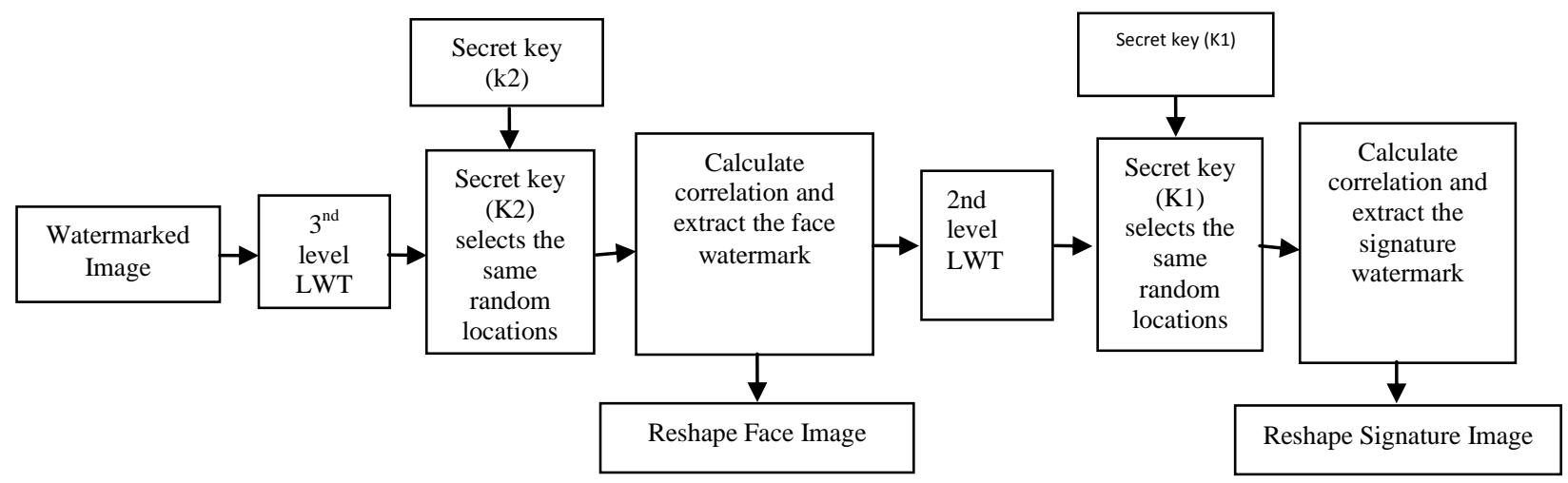

Fig 4: Block Diagram of proposed watermarking extraction scheme.

\section{EMBEDDING FACTOR OPTIMIZATION}

The embedding factor $\boldsymbol{k}$ is of vital significance to any watermarking scheme. Its value has a direct implication on the following two factors

1) Robustness: As $k$ increases, the algorithm becomes more resilient to attacks and hence more robust.

2) Imperceptibility: As $k$ increases, the image becomes more distorted and hence imperceptibility is not achieved.

Due to this trade-off, a multi objective optimization technique is used to select appropriate strength factor so as to maintain imperceptibility with improved attack resilience. To show the effect of $k$ on image distortions, the performance evaluation of the method has been done using image quality determination metric Structural Similarity Index Measure (SSIM) [16]. While PSNR is a commonly used metric, SSIM index is an advanced method for measuring the similarity between two images. SSIM is designed to improve on traditional methods like PSNR and MSE by modelling image distortion as a combination of three factors considering the properties of Human Visual System (HVS). These three factors are loss of correlation, luminance distortion and contrast distortion.

Let $I_{\text {original }}=x$ be the original image and $I_{w m}=y$ be the manipulated (either watermarked or attacked image), the SSIM quality index is defined as$$
\operatorname{SSIM}(x, y)=
$$$$
\frac{\left(2 \mu_{x} \mu_{y}+C_{2}\right)\left(2 \sigma_{x y}+C_{2}\right)}{\left(\mu_{x}^{2}+\mu_{y}{ }^{2}+C_{2}\right)\left(\sigma_{x}^{2}+\sigma_{y}^{2}+C_{2}\right)}
$$$$
\mu_{x}, \mu_{y}, \sigma_{x}{ }^{2}, \sigma_{y}{ }^{2} \text { are the mean value and variances of } x \text { and }
$$$$
y \text { respectively and }
$$$$
\sigma_{x y}=\frac{1}{N-1} \sum_{i=1}^{N}\left(x_{i}-\mu_{x}\right)\left(y_{i}-\mu_{y}\right)
$$

$N=$ Number of samples. Parameters $C_{1}$ and $C_{2}$ are defined as

$C_{1}=\left(K_{1} l\right)^{2} \quad, C_{2}=\left(K_{2} l\right)^{2}$

$l$ is the dynamic range of the pixel values(for gray scale images it ranges from 0-255) and $K_{1}, K_{2}<<1$ are small
}

constants. The standard value for $K_{1}$ is taken as 0.01 while for $K_{2}$, it is 0.03 . The image perceptual comparison is best when the size of $x$ and $y$ is the same.In practice, one usually requires a single overall quality measure of the entire image. We use a mean SSIM (MSSIM) index to evaluate the overall image quality.

$M S \operatorname{SIM}=\frac{1}{M} \sum_{j=1}^{M} \operatorname{SSIM}\left(x_{j}, y_{j}\right)$

Thus the parameter optimization will be achieved using two objective functions $f_{i}(k)=1-\operatorname{SSIM}(k)$ and $f_{r}(k) . f_{i}(k)$ shows the effect of $k$ on imperceptibility while $f_{r}(k)$ shows the impact of $k$ on attack resilience and is calculated as the error probability function described as

$P_{\text {error }}=\frac{1}{2} P_{\text {error } \mid 0}+\frac{1}{2} P_{\text {error } \mid 1}$

Both the functions have a contrasting nature while the imperceptibility increases monotonically with $k$, robustness is monotonically decreasing. Thus the aim is to find an optimum value of $k$ so as to minimize both the functions. The goal attainment method given in [18] provides a solution to find optimum $k$ value according to SSIM which gives satisfactory level of attack resilience. In this approach the value $0.5<k<0.7$ is found to be optimal for most of the images.

\section{EXPERIMENTAL RESULTS AND DISCUSSIONS}

\subsection{Experimental Setup}

It is important to evaluate an image watermark algorithm on many different images. Images should cover a broad range of contents and types. The proposed technique has been tested on standard evaluation images for watermarking algorithms. To evaluate the performance of the proposed watermarking algorithm, MATLAB platform is used. The standard Yale database for 50 face images has been used and a signature database consisting of signatures of 50 users has been used for experimental purposes.

Fig 5 shows the original image as well as watermarked image and the original as well as extracted watermarks. In all 
the above images, the same face and signature image has been used as a watermark.
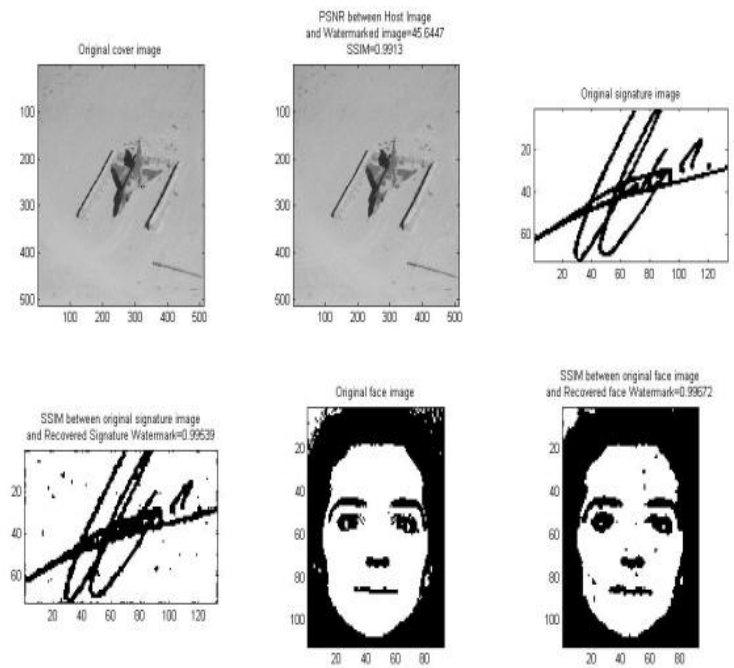

(a)
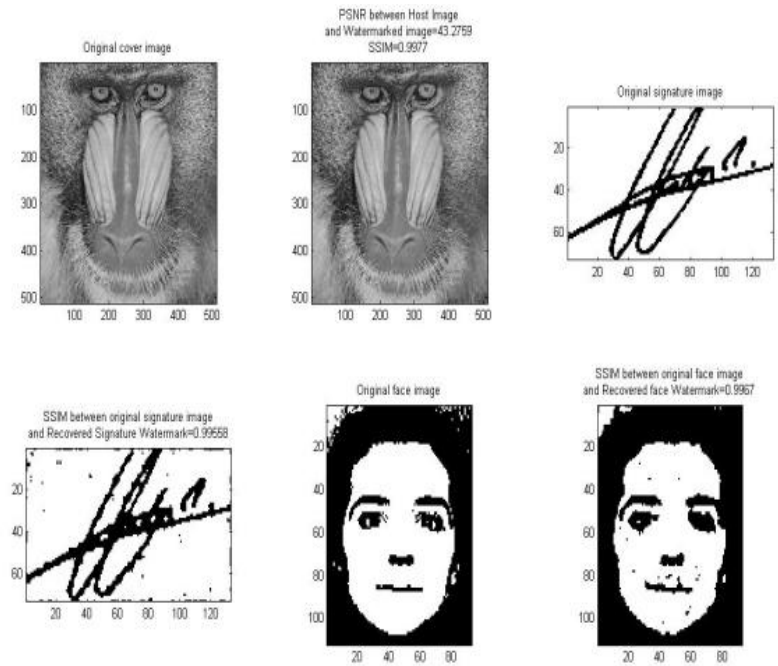

(b)
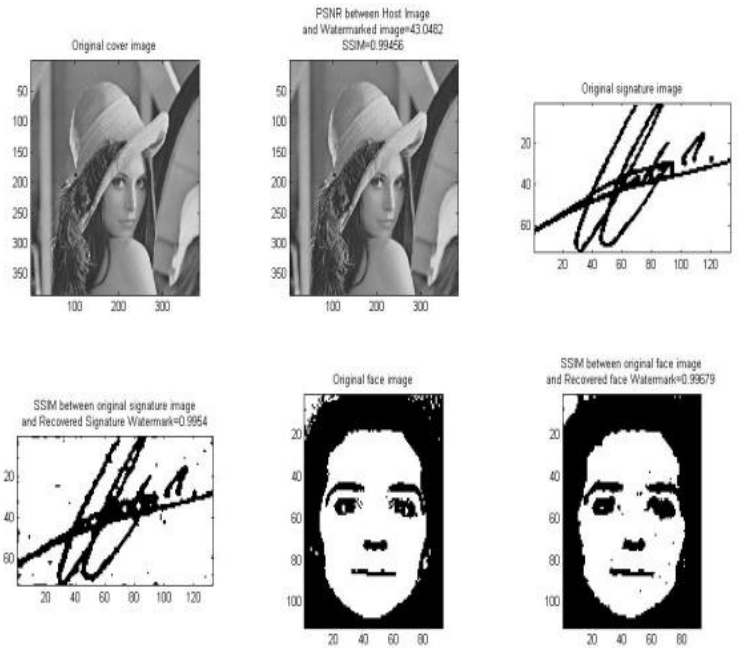

(c)
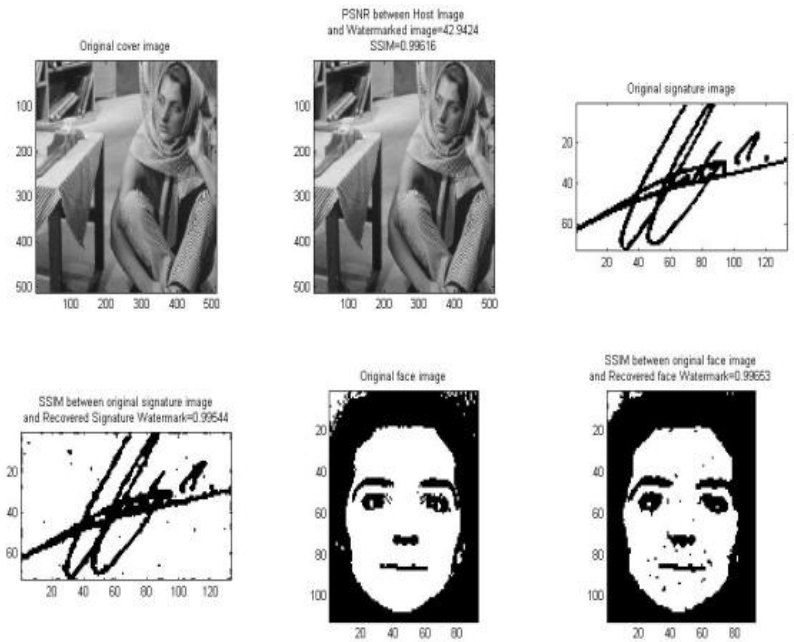

(d)
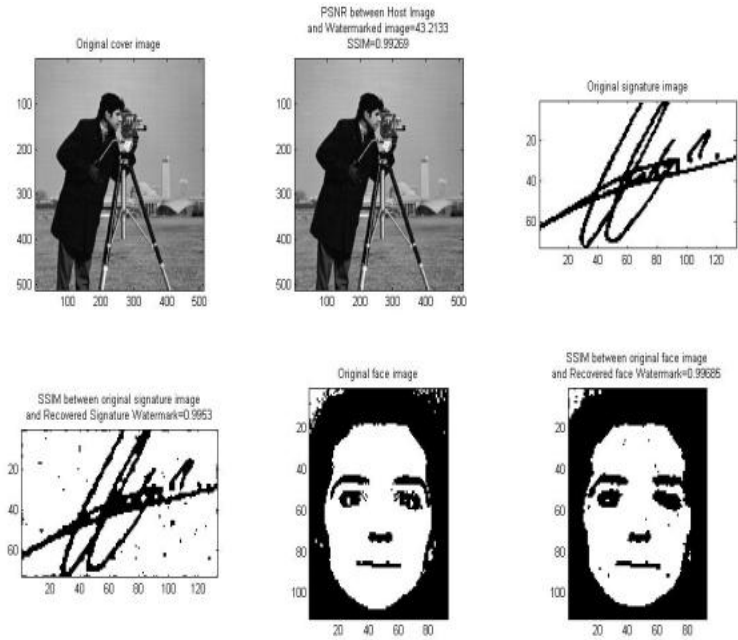

(e)
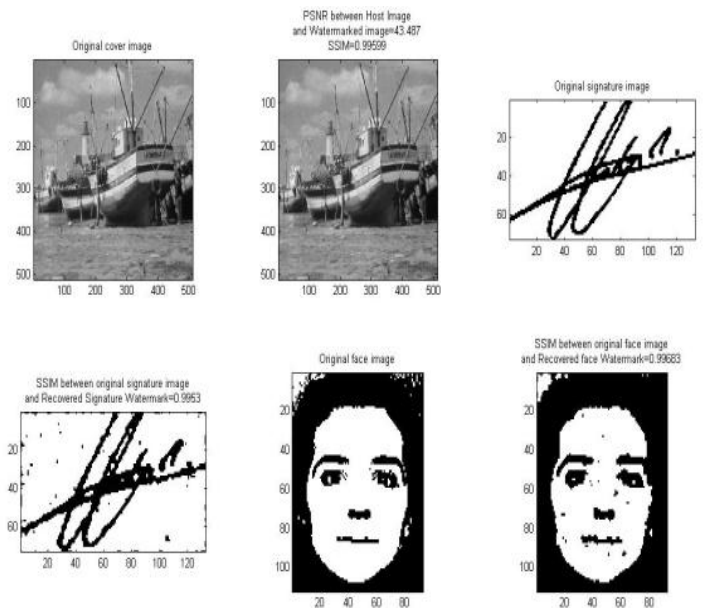

(f)

Fig 5: The original and recovered face and signature watermark after applying the embedding and extraction algorithm on standard test image using the same signature and face image (a) Plane (b) Baboon (c) Lena (d) Barbara (e) Cameraman (f) Boat. 


\subsection{Robustness Tests under StirMark}

\section{Attacks}

The robustness of the proposed algorithm has been tested against the benchmark StirMark [17] attack. These attacks include common signal processing attacks like median filtering, Gaussian noise addition, Jpeg Compression, or geometric attacks like resizing, rotation or a combination of both.

\subsubsection{Median filtering}

The most common signal processing attack in digital image is filtering. Table I shows the MSSIM values for the extracted face and signature watermarks, after applying median filter of varying filter lengths. It can be observed that even after the degradations caused in the images, the recovered watermarks are very much recognizable. Some results for the same are shown in fig 6 .

Table I: MSSIM results of extracted watermarks Median Filtering with different filter sizes attack

\begin{tabular}{|c|c|c|c|c|c|c|}
\hline & \multicolumn{6}{|c|}{$3 \times 3$} \\
Image & Face & Signature & Face & Sign & Face & Sign \\
\hline & & & & & & \\
\hline Barbara & 0.99748 & 0.99568 & 0.99746 & 0.99609 & 0.99749 & 0.99583 \\
& & & & & & \\
\hline Lena & 0.99644 & 0.99536 & 0.99644 & 0.99518 & 0.99646 & 0.99539 \\
\hline Boat & 0.99636 & 0.99627 & 0.99616 & 0.99578 & 0.99627 & 0.99631 \\
\hline Plane & 0.99742 & 0.99632 & 0.99748 & 0.9965 & 0.99752 & 0.99605 \\
\hline Baboon & 0.99618 & 0.99512 & 0.99616 & 0.99509 & 0.99604 & 0.99511 \\
\hline
\end{tabular}
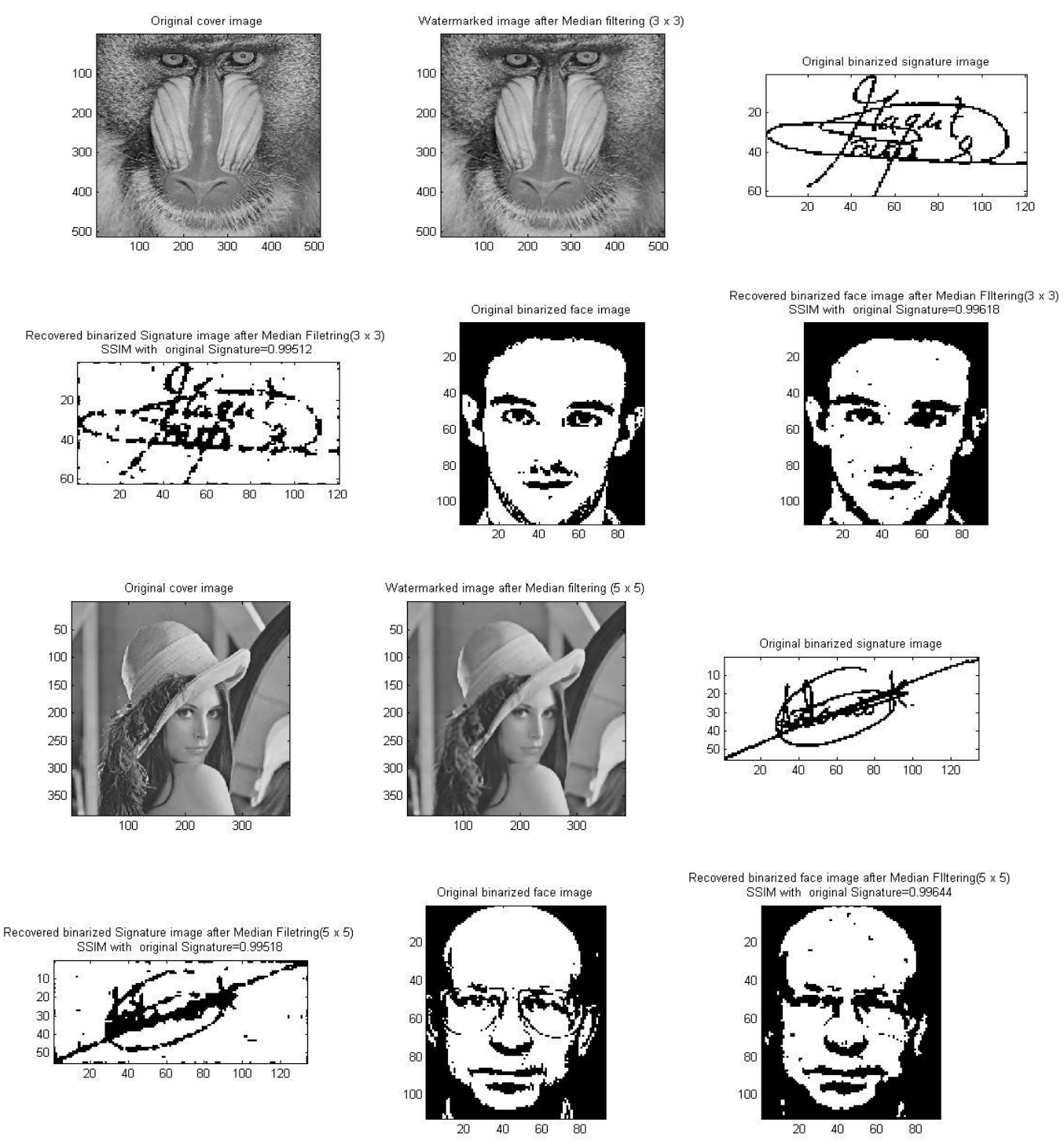

Fig 6: Extracted signature and face watermarks after median filtering on different images. 
Volume 72-No.9, May 2013

\subsubsection{Gaussian noise}

Noise is added to image to degrade its quality. Robustness against additive Gaussian noise is estimated by degrading the watermark image by randomly adding
Gaussian noise with mean 0 and variance 0.01 . Table II shows the MSSIM values for various recovered watermarks while fig 7 shows the results pictorially.

Table II: MSSIM results of extracted watermarks Under Gaussian filtering Attack mean=0, variance=0.01

\begin{tabular}{|c|c|c|}
\hline \multirow{2}{*}{ Image } & \multicolumn{2}{|c|}{ Gaussian filtering } \\
\hline & Face & Signature \\
\hline Barbara & 0.99671 & 0.99514 \\
\hline Lena & 0.99753 & 0.99578 \\
\hline Boat & 0.99597 & 0.99544 \\
\hline Plane & 0.99753 & 0.99578 \\
\hline Baboon & 0.99747 & 0.99512 \\
\hline
\end{tabular}
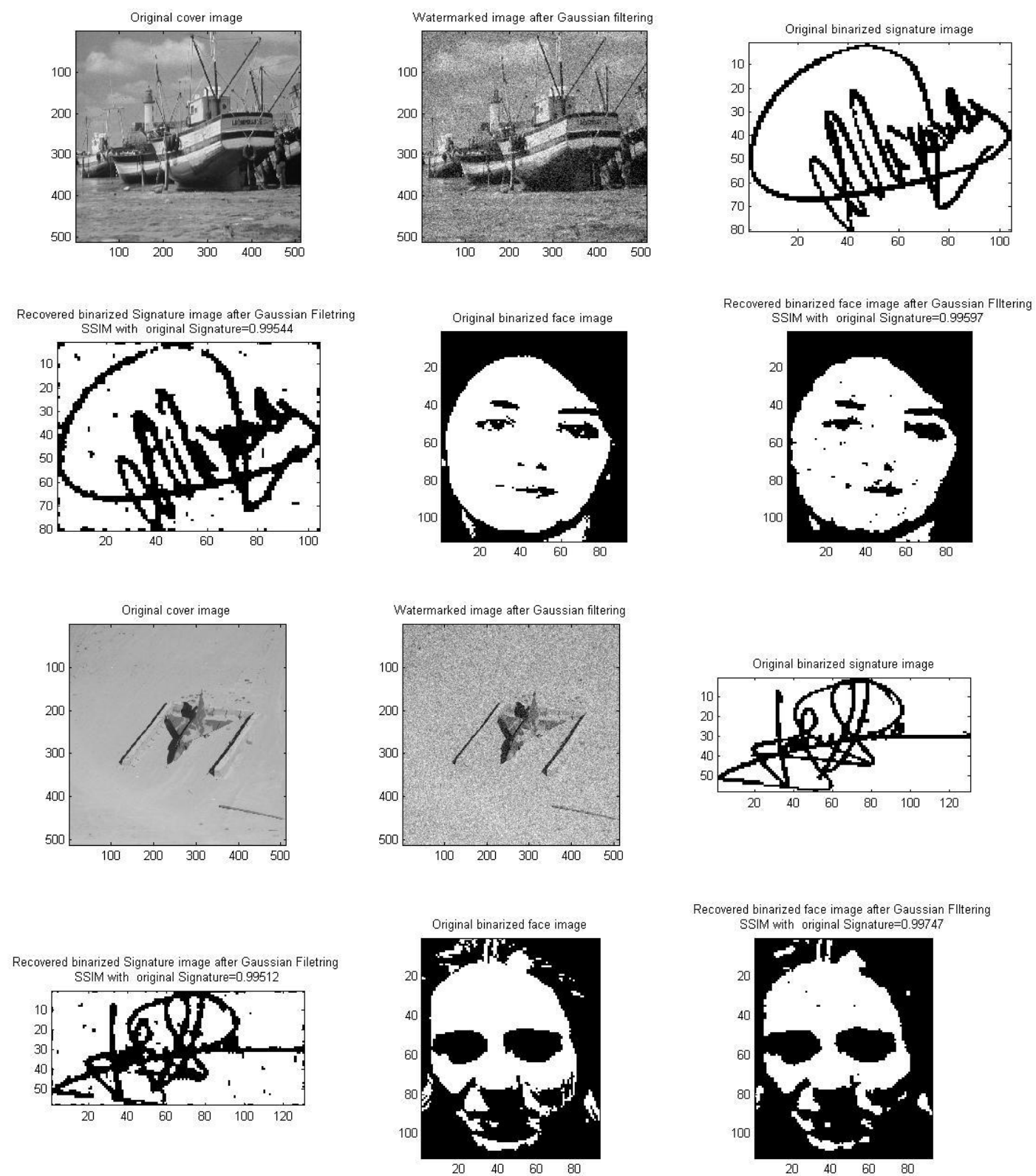

Fig 7: Extracted watermarks after addition of Gaussian noise to watermarked image 


\subsubsection{Scaling}

The images were either cropped or resized.
Table III and fig 8 present the results of the effect of various scaling factors on the watermarked images.

Table III: MSSIM results of extracted watermarks under scaling Attack

\begin{tabular}{|c|c|c|c|c|c|c|c|c|}
\hline \multirow{2}{*}{ Image } & \multicolumn{9}{|c|}{ Scaling Factor } \\
& \multicolumn{2}{|c|}{0.5} & \multicolumn{2}{c|}{0.75} & 1.5 & S \\
\hline & Face & Signature & Face & Signature & Face & Signature & Face & Signature \\
\hline Barbara & 0.99682 & 0.99485 & 0.99648 & 0.99552 & 0.9965 & 0.99528 & 0.99697 & 0.99503 \\
\hline Lena & 0.99597 & 0.99539 & 0.99629 & 0.99619 & 0.99619 & 0.99569 & 0.99605 & 0.99535 \\
\hline Boat & 0.99735 & 0.99523 & 0.99723 & 0.99741 & 0.99741 & 0.99587 & 0.99759 & 0.99566 \\
\hline Plane & 0.99665 & 0.99526 & 0.99674 & 0.9966 & 0.9966 & 0.99505 & 0.9967 & 0.99547 \\
\hline Baboon & 0.99562 & 0.99643 & 0.99595 & 0.99587 & 0.99587 & 0.99648 & 0.99583 & 0.99723 \\
\hline
\end{tabular}

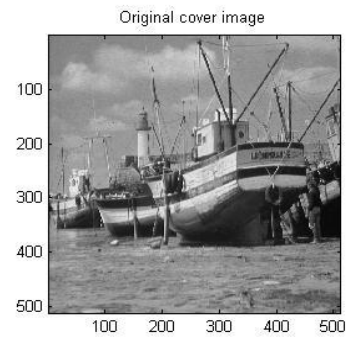

Recovered binarized Signature image after scaling
SSIM with original Signature $=0.99579$
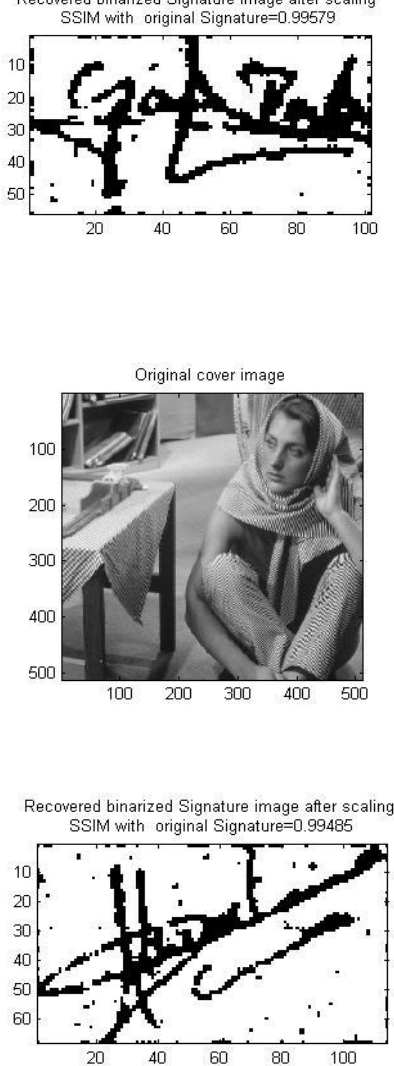
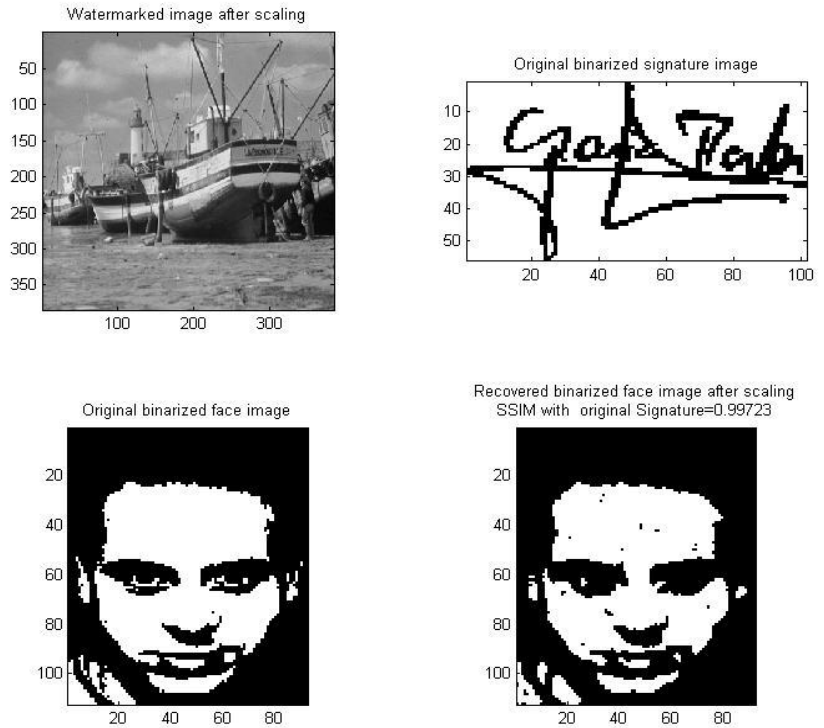

(a)
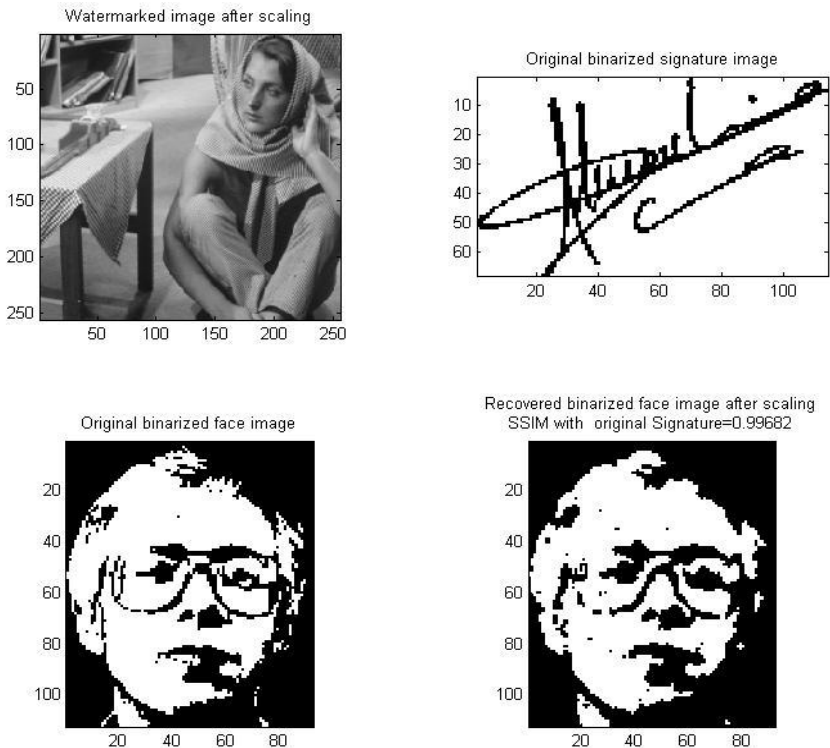

(b) 

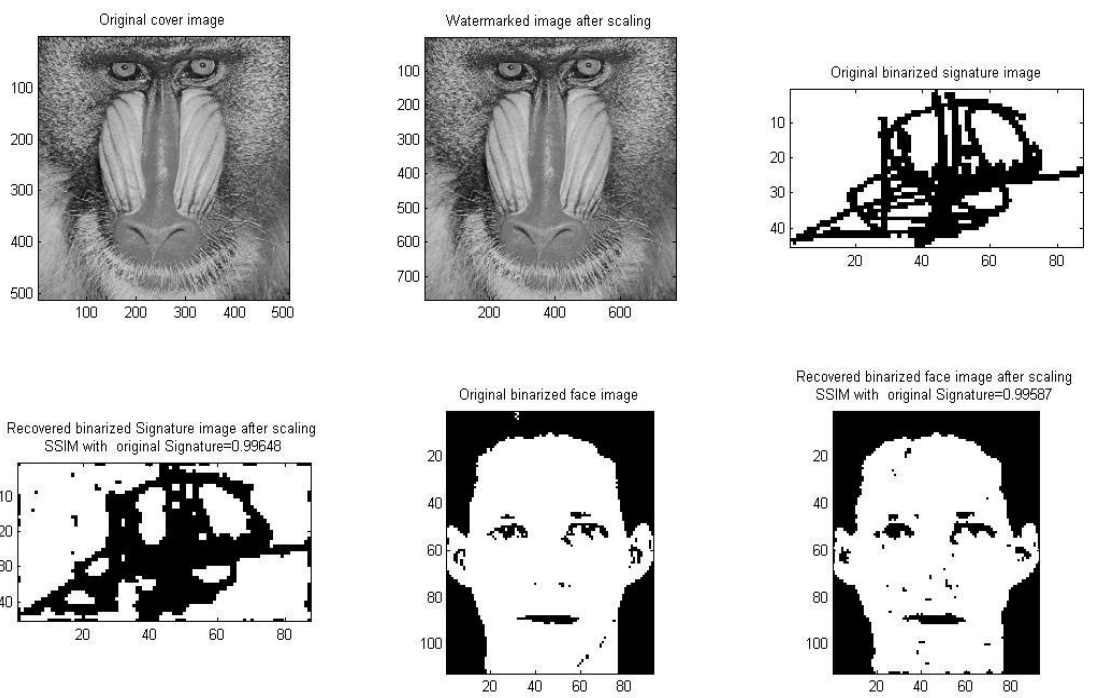

(c)
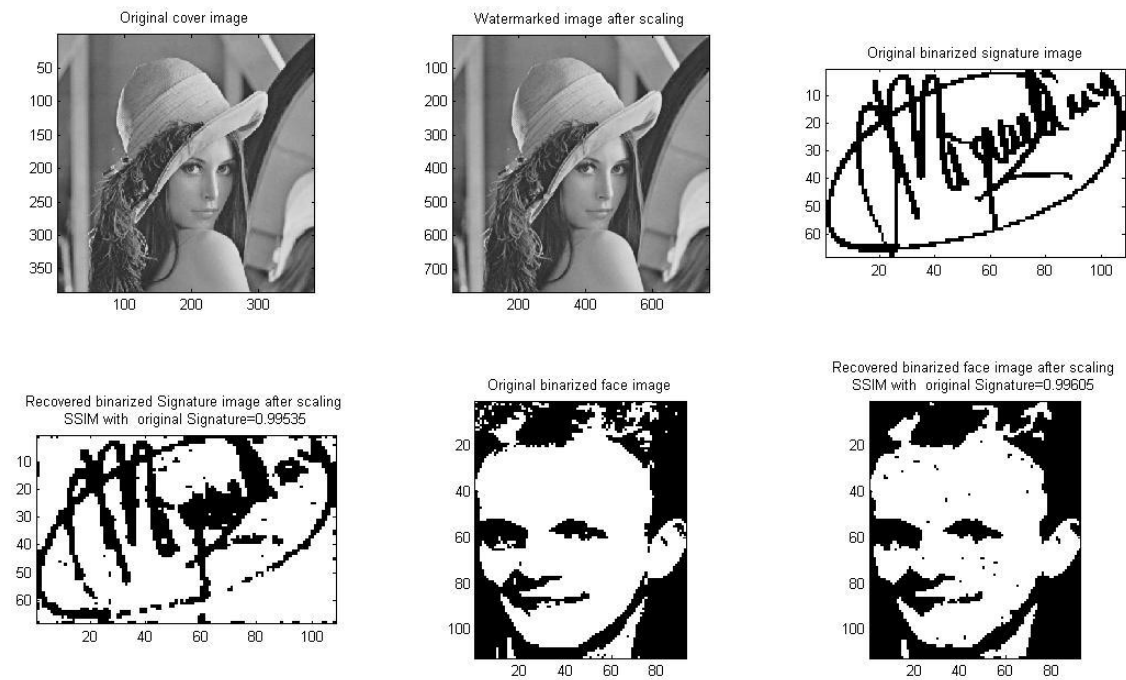

(d)

Fig 8: Effect of various scaling factors on the recovered watermarks (a) 0.5 (b) 0.75 (c) 1.5 (d) 2.0

\subsubsection{JPEG Compression}

Another most common manipulation in digital image is image compression. To check the robustness against Image Compression, the watermarked image is compressed using
JPEG compression for different compression ratios. MSSIM values for the extracted watermarks are depicted in Table IV and subsequently in fig 9 .

Table IV: MSSIM results for JPEG Compression with varying Q-factor

\begin{tabular}{|c|c|c|c|c|c|c|c|c|c|c|}
\hline \multirow[t]{2}{*}{ Image } & \multicolumn{3}{|c|}{20} & \multicolumn{2}{|c|}{40} & \multicolumn{2}{|l|}{60} & \multicolumn{2}{|l|}{0} & 00 \\
\hline & Face & Signature & Face & Signature & Face & Signature & Face & Signature & Face & Signature \\
\hline Barbara & 0.99593 & 0.99552 & 0.999596 & 0.99568 & 0.99587 & 0.99567 & 0.99591 & 0.99546 & 0.99594 & 0.99557 \\
\hline Lena & 0.99739 & 0.99614 & 0.99743 & 0.99592 & 0.99738 & 0.99613 & 0.99736 & 0.99636 & 0.99736 & 0.99612 \\
\hline Boat & 0.99743 & 0.99695 & 0.9976 & 0.99684 & 0.9976 & 0.99674 & 0.99758 & 0.99671 & 0.99756 & 0.99663 \\
\hline Plane & 0.9972 & 0.99476 & 0.99714 & 0.99495 & 0.99714 & 0.99504 & 0.99708 & 0.99474 & 0.99713 & 0.99512 \\
\hline Baboon & 0.99631 & 0.99545 & 0.99621 & 0.99521 & 0.99624 & 0.99521 & 0.99619 & 0.99516 & 0.9961 & 0.99517 \\
\hline
\end{tabular}



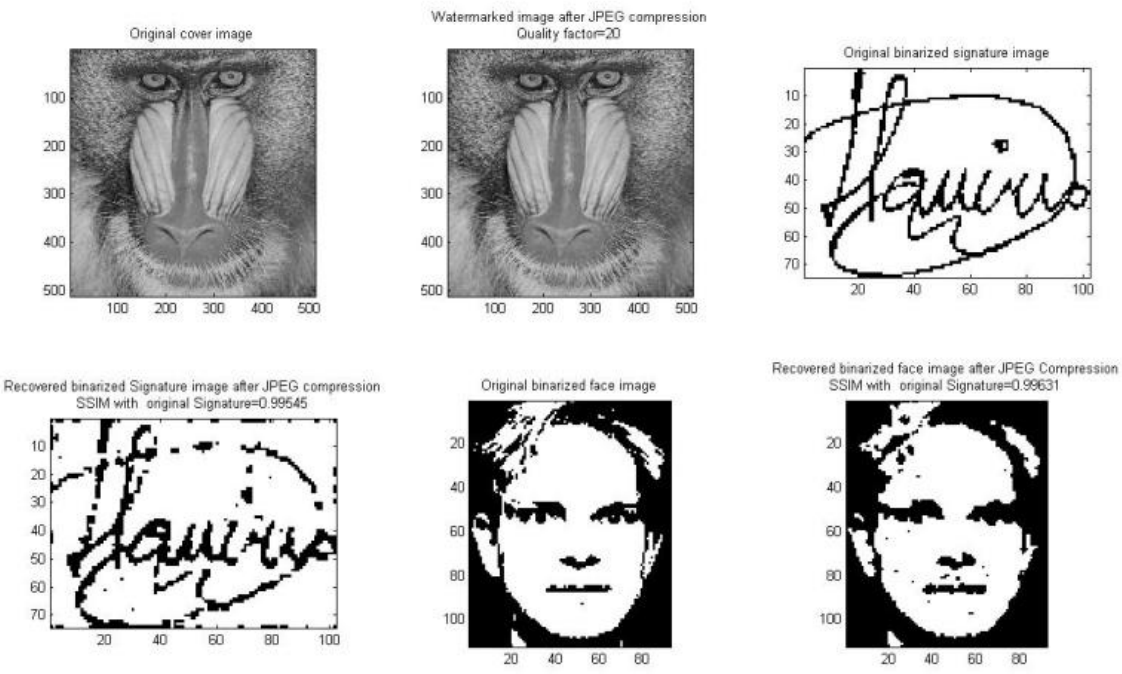

(a)
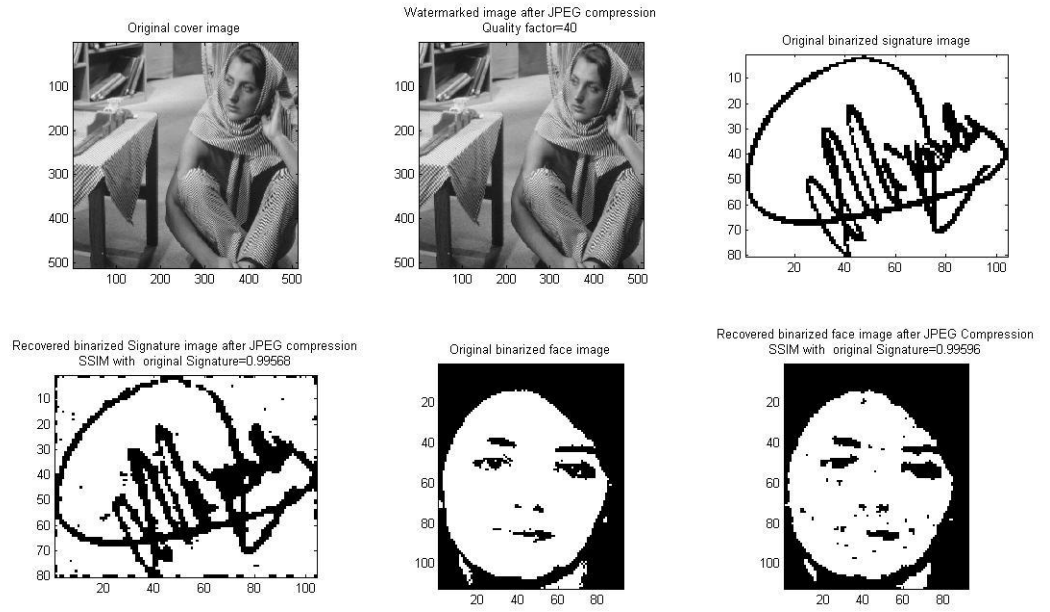

(b)

Fig 9: Extracted watermarks after JPEG Compression (a) Q-factor $=20$ (b) Q- factor=40

\subsubsection{Rotation}

In the next experiment, the robustness of the proposed algorithm is tested against rotation attack. The proposed embedding approach is robust to rotation provided that one can compensate for the loss of synchronization. Table $\mathrm{V}$ and fig 10 shows the MSSIM values after the watermarked image was rotated in the range of ( -5 degree to 5 degree).

\subsubsection{Combined Attacks}

In addition to the common individual attacks, the robustness of the algorithm was also tested against the combined attacks. The results of the same are presented in table VI. 
Table V: MSSIM results of extracted watermarks under rotation attack

\begin{tabular}{|c|c|c|c|c|c|c|c|c|}
\hline \multirow{3}{*}{ Image } & \multicolumn{8}{|c|}{ Rotation Angle (Degrees) } \\
\hline & \multicolumn{3}{|c|}{-5} & -1 & \multicolumn{2}{|c|}{1} & & \\
\hline & Face & Signature & Face & Signature & Face & Signature & Face & Signature \\
\hline Barbara & 0.99744 & 0.99512 & 0.9974 & 0.99501 & 0.99719 & 0.99469 & 0.99735 & 0.99484 \\
\hline Lena & 0.99659 & 0.99454 & 0.99613 & 0.99456 & 0.99641 & 0.99477 & 0.99624 & 0.9949 \\
\hline Boat & 0.99669 & 0.99522 & 0.99661 & 0.99566 & 0.9964 & 0.99534 & 0.99666 & 0.99492 \\
\hline Plane & 0.99705 & 0.99524 & 0.99711 & 0.99537 & 0.99697 & 0.99515 & 0.99696 & 0.99459 \\
\hline Baboon & 0.99729 & 0.99553 & 0.99705 & 0.99539 & 0.99721 & 0.99512 & 0.99712 & 0.99499 \\
\hline
\end{tabular}
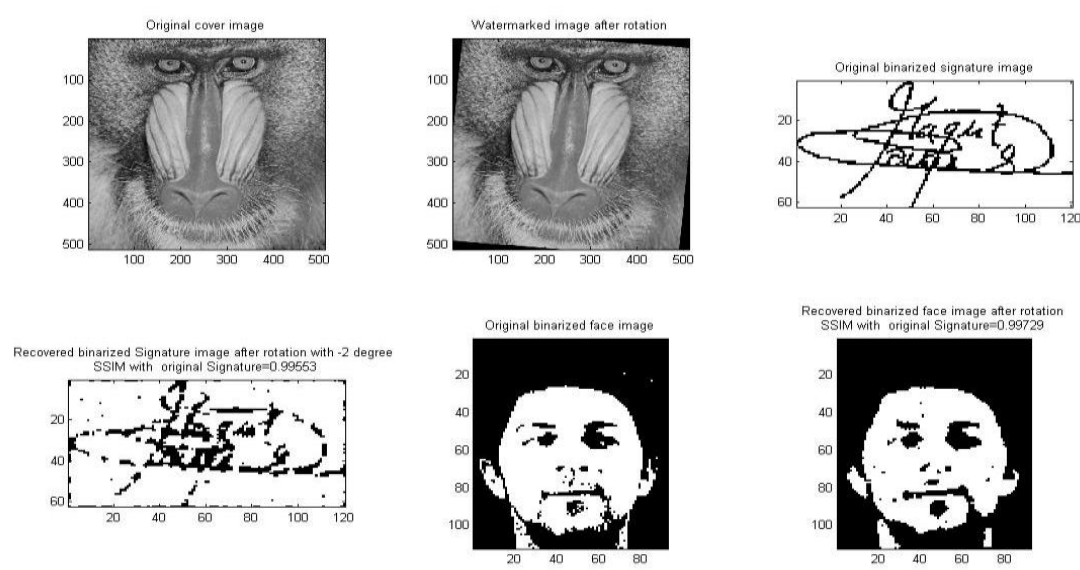

(a)
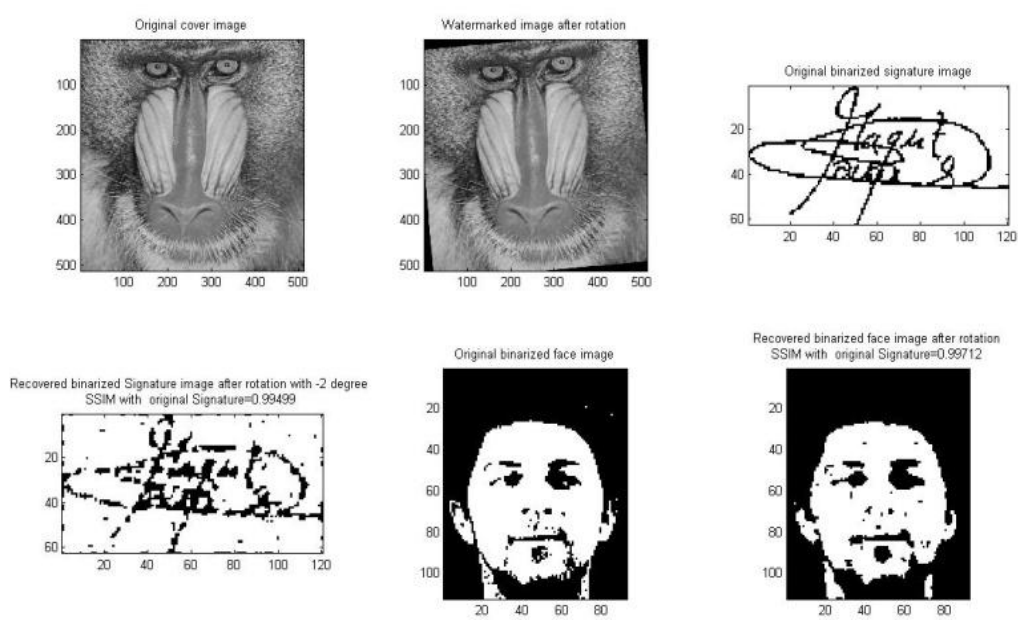

(b)

Fig 10: Extracted watermarks after (a) -5 degree rotation (b) 5 degrees rotation 
Table VI: MSSIM results of extracted watermarks under Combined Attacks

\begin{tabular}{|c|c|c|c|c|c|c|c|c|c|c|}
\hline & \multicolumn{9}{|c|}{ Barbara } & \multicolumn{9}{c|}{ Boat } & Lena \\
\hline & Face & Sign & Face & Sign & Face & Sign & Face & Sign & Face & Sign \\
\hline $\begin{array}{c}\text { JPEG_20+ } \\
\text { Scaling(0.5) }\end{array}$ & 0.99584 & 0.99669 & 0.99682 & 0.99539 & 0.99618 & 0.99571 & 0.9963 & 0.99581 & 0.99702 & 0.99505 \\
\hline $\begin{array}{c}\text { JPEG_20+ } \\
\text { Scaling(2.0) }\end{array}$ & 0.99583 & 0.99723 & 0.99697 & 0.99503 & 0.99759 & 0.9566 & 0.99605 & 0.99535 & 0.9967 & 0.99547 \\
\hline $\begin{array}{c}\text { JPEG_20+ } \\
\text { Med_filt } \\
\text { (3x3) }\end{array}$ & 0.99618 & 0.99512 & 0.99748 & 0.99568 & 0.99636 & 0.99627 & 0.99644 & 0.99536 & 0.99742 & 0.99632 \\
\hline $\begin{array}{c}\text { JPEG_20+ } \\
\text { Med_filt } \\
\text { (5x5) }\end{array}$ & 0.99616 & 0.99509 & 0.99746 & 0.99609 & 0.99628 & 0.9963 & 0.99644 & 0.99518 & 0.99752 & 0.99608 \\
\hline $\begin{array}{c}\text { JPEG_20+ } \\
\text { Med_filt } \\
\text { (7x7) }\end{array}$ & 0.99604 & 0.99511 & 0.99749 & 0.99583 & 0.99627 & 0.99631 & 0.99646 & 0.99539 & 0.99752 & 0.99605 \\
\hline $\begin{array}{c}\text { JPEG_20+ } \\
\text { Gaussian } \\
\text { noise }\end{array}$ & 0.99769 & 0.99487 & 0.99644 & 0.99581 & 0.99613 & 0.99581 & 0.99758 & 0.996 & 0.99733 & 0.99546 \\
\hline
\end{tabular}

\section{CONCLUSION}

The paper presents a novel biometric multimodal watermarking algorithm using LWT. The watermark is embedded into the high frequency areas of the cover image using Watson's HVS criterion. Watermark detection is done using the correlation detector with the help of biorthogonal wavelets to make the recovery of the watermark better.

A delicate balance has been maintained in the trade-off between imperceptibility and robustness through the multiobjective optimization approach.

The robustness of the proposed method has been experimentally validated using the standard test benchmarks and the results are far more superior than any approach used for multimodal biometric embedding. The work can be further extended by incorporating pattern algorithms for the purpose of authentication of the extracted watermark.

\section{REFERENCES}

[1] Anil K Jain, Umut Uludag, "Multimedia Content protection via Biometric based Encryption," Proc. of IEEE International Conference on Multimedia and Expo, ICME, July 2003.

[2] Kundur D. and D. Hatzinakos, "Digital Watermarking using Multi Resolution Wavelet Decomposition," Proc. of IEEE International Conference on Acoustics, Speech and Signal Processing, vol. 5, pp. 2969-2972, May 1998.

[3] Yang S.H., "Wavelet Filter Evaluation for Image Watermarking," Proc. of IEEE International Conference on Acoustics, Speech and Signal Processing, vol. 3, pp. 525-528, April 2003.

[4] Marusic S., D.B. Tay, G. Deng, and M. Palaniswami, "Even-length Biorthogonal Wavelets for Digital Watermarking," Proc. of Eighth International Symposium on Signal Processing and its Applications, vol. 1, pp. 17-20, August 2005.

[5] Pla O.G., E.T. Lin, and E.J. Delp, "A Wavelet Watermarking Algorithm Based on a Tree Structure," Proc. of SPIE-IS\&T Electronic Imaging, vol. 5306 , pp. 571-580, 2004.

[6] Mayank Vasta, Richa Singh, Afzel noore ,"Improving Biometric Recognition Accuracy and Robustness using DWT and SVM watermarking," IEICE Electronics Express, vol 2, pp.362-367, June 2005.
[7] Cheng-Yaw Low, Andrew Beng-Jin Teoh, Connie Tea, “ Fusion of LSB and DWT Biometric Watermarking for Offline Handwritten Signature," Proc. of 2008 Congress on Image and Signal processing, vol. 5, pp.702-708, May 2008.

[8] A. M. Namboodiri and A. K. Jain, "Multimedia Document Authentication using On-line Signature as Watermarks," Proc. of the International Society for Optical Engineering (SPIE), vol. 5306, pp. 653-662, June 2004.

[9] M. Barni, F. Bartolini, A. Piva, "Improved Wavelet based Watermarking through Pixel Wise Masking," IEEE Transactions on Image Processing vol. 10, pp. 783-791, May 2001.

[10] A.A. Reddy, B.N. Chatterji, "A New Wavelet Based Logo-Watermarking Scheme," Pattern Recognition Letters, vol. 26, pp. 1019-1027, May 2005.

[11] W. Sweldens, "The Lifting Scheme: A Construction of Second Generation Wavelets," SIAM Journal on Mathematical Analysis, vol. 29, pp. 511-546, March 1998.

[12] Fritz Keinert, "Biorthogonal Wavelets for Fast Matrix Computations," Applied and Computational Harmonic Analysis, vol. 1, pp. 147-156, March 1994.

[13] Cox J. Kilian, F. Leighton, and T. Shamoon, "Secure Spread Spectrum Watermarking for Multimedia," IEEE Transaction on Image Processing, vol. 6, pp. 1673-1687, Dec. 1997.

[14] Meenakshi S Arya and Rajesh Siddavatam, "A Novel Biometric Watermarking Approach Using LWT- SVD," Communications in Computer and Information Science, Information Technology and Mobile Communication, vol. 147, pp. 123-131, April 2011.

[15] Piotr Porwik, "The Compact Three Stages Method of the Signature Recognition," Proc. of 6th International Conference on Computer Information Systems and Industrial Management Applications, pp. 282-287, June 2007.

[16] Zhou Wang, Alan Conrad Bovik, Hamid Rahim Sheikh and Eero P. Simoncelli, "Image Quality Assessment: From Error Visibility to Structural Similarity," IEEE Transactions on Image Processing, vol. 13, pp. 600- 612, April 2004. 
[17] F. A. P. Petitcolas, "Watermarking Schemes Evaluation," IEEE Signal Processing Magazine, vol. 17, pp. 58-64, Sep. 2000.
[18] F. Gembicki and Y. Haimes, "Approach to Performance and Sensitivity Multiobjective Optimization: The Goal Attainment Method," IEEE Transactions on Autom. Control, vol. 20, pp. 769-771, Dec. 1975. 